\title{
Sympathetic cooling of molecular ions with ultracold atoms
}

\author{
Eric R. Hudson
}

\section{Correspondence:}

eric.hudson@ucla.edu

Department of Physics and

Astronomy, UCLA, Los Angeles, CA, 90095, USA

\section{Springer}

\begin{abstract}
Sympathetic cooling of molecular ions with ultracold gases is enabling a new era of research in chemistry and physics. There has been much progress in this new field in the last several years and many unanticipated challenges have been overcome. The aim of the present manuscript is to provide a concise review of this work and discuss the way forward for the field.
\end{abstract}

Keywords: Cold molecular ions, Sympathetic cooilng, Atom-ion chemistry, Nonequlibrium physics

\section{Review}

\section{Introduction}

The ability to produce atoms and atomic ions in a single quantum state has revolutionized atomic physics [1] and the technologies it provides [2-5]. To produce this quantum matter, the temperature of the atoms and ions are lowered such that the Gibbs factor is appreciable for only the quantum ground state. The techniques for reaching these, socalled, ultracold temperatures, which are the subject of several recent Nobel prizes, are centered around laser cooling [6].

In laser cooling, the simple electronic structure of an atomic system is exploited to remove thermal energy from a particle by scattering a large number of photons from it. If the ability to prepare single quantum state matter, i.e. ultracold matter, could be extended to more complicated systems, e.g. molecules, a new era in both physics and chemistry, and the technologies they provide, will begin $[7,8]$. Unfortunately, the same complexity that makes molecules attractive renders them unamenable, with few exceptions [9-11], to laser cooling.

An interesting alternative to laser cooling for the production of a single-quantum-state molecular sample, is to use a reservoir of ultracold atoms to sympathetically cool the molecules to ultracold temperatures [12-14] - in much the same way that cryogenic systems use a helium buffer gas to cool other objects to cryogenic temperatures. In principle, this technique of ultracold-atom sympathetic cooling is completely general since it relies only on the second law of thermodynamics. In reality, however, there are many practical issues that must be considered before efficient sympathetic cooling of molecules by ultracold atoms can occur. These issues and the means to understand and control them are the subject of this review. This manuscript is an attempt to concisely describe the current

(c) The Author(s). 2016 licensee Springer on behalf of EPJ. This is an Open Access article distributed under the terms of the Creative Commons Attribution License (http://creativecommons.org/licenses/by/4.0), which permits unrestricted use, distribution, and reproduction in any medium, provided the original work is properly credited. 
understanding of these practical issues in the so-called MOTion trap system and the solutions we have implemented in our laboratory to circumvent them. As with all things, there are many exceptions to the 'rules' presented here, which have been omitted for the sake of brevity. Likewise, other exciting techniques for producing ultracold molecular ions, such as sympathetic cooling with atomic ions in combination with rovibrational optical pumping [15-17] or state-selective ionization [18], and cooling in multipole traps [19], are not reviewed here for the same reason. In what follows, the MOTion trap system is first introduced, sympathetic cooling with ultracold atoms discussed, and techniques for creation and detection of molecular ions are summarized.

\section{The MOTion trap}

The first challenge to be addressed is the design of the experimental system that will enable sympathetic cooling of molecules by ultracold atoms. This system must be capable of immersing the desired molecules in the ultracold atom cloud (typically a spherical volume of $\sim 0.01-1 \mathrm{~cm}^{3}$ ), so they can be sympathetically cooled. Since most methods of producing gas-phase molecules in vacuum lead to approximately room temperature, or hotter, molecules it is desirable to construct a trap with a potential energy depth of the same order. Further, because sympathetic cooling is largely species independent, it is desirable that the trapping potential is also species (and quantumstate) independent. These two factors combined suggest that sympathetic cooling with ultracold atoms is particulary effective for cooling of charged molecules, which can be trapped with ion trapping technology [20], yielding a large and state-independent trap depth.

Generally speaking, the ultracold atoms available for current experiments are produced by laser cooling [6] and will therefore either be in the magneto-optical trap (MOT) that produced them or a secondary, e.g. magnetic or dipole, trap following laser cooling [21]. Because laser-cooled atom clouds are small, they have limited heat capacity and therefore limited cooling power. Further, as will be discussed later, the use of an ion trap introduces a small, continuous heat load on the molecules. Therefore, for efficient sympathetic cooling of molecular ions it appears that the use of a MOT, where the atomic temperature is held constant by active laser cooling, is a good choice for the ultracold atom reservoir; otherwise, the molecular ions heat the trapped, non-actively cooled sample of ultracold atoms, complicating the production of ultracold molecular ions.

As a result, we have built a combination MOT and ion trap, dubbed the MOTion trap, as shown in Fig. 1. The choice of a linear Paul trap is driven by the simplicity and large optical access of the design. In this figure, $\mathrm{Yb}^{+}$ions are laser-cooled and imaged in the ion trap as a substitute for the molecular ions to allow visualization of the trapped ion cloud by laser induced fluorescence imaging. While the MOTion trap was proposed as a means to provide ultracold molecular ions, it has also produced many important results in quantum chemistry since it brings ultracold atoms and ions together in a controllable way. Specifically, because ion trapping provides an effectively infinite trap lifetime, simple reaction product detection, and the ability to work with very rare species, a variety of chemical studies, not currently possible for all neutral reactions have been carried out. The interested reader is directed to the excellent recent reviews of Refs. [7, 22]. 


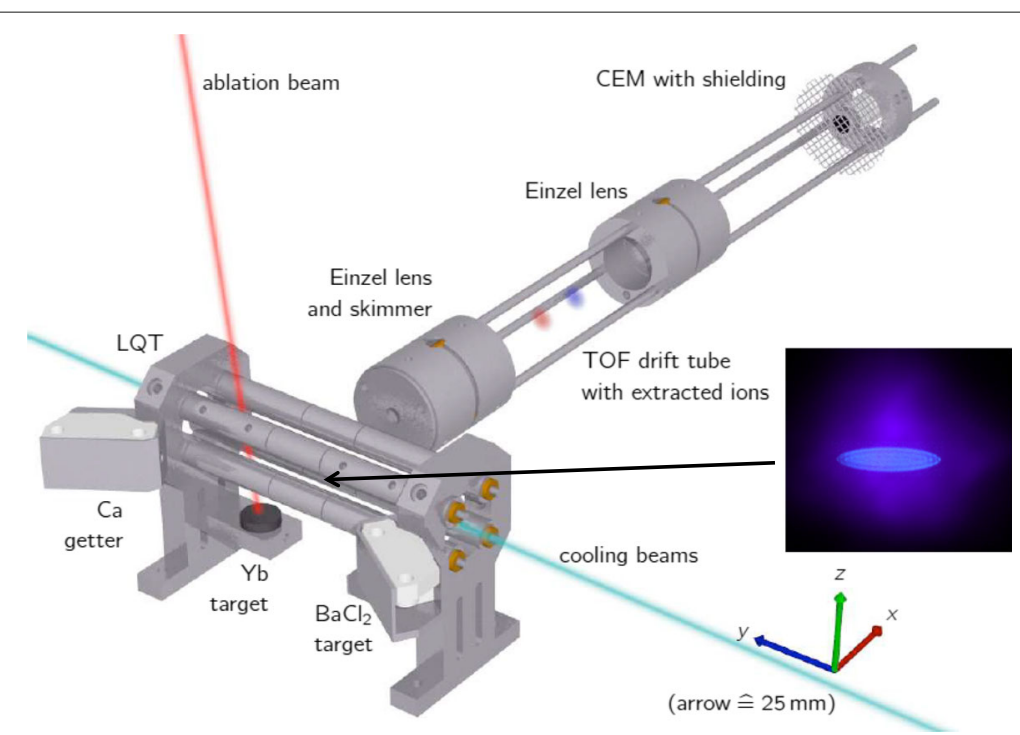

Fig. 1 Schematic of the MOTion trap and the radially coupled time-of-flight mass spectrometer. Also shown as an inset is an image of trapped $\mathrm{Yb}^{+}$ions immersed in the Ca MOT

\section{Sympathetic cooling in the MOTion trap}

At first glance, sympathetic cooling appears an ideal method for producing ultracold molecular ions. Simply place the molecules in a cold bath and let them equilibrate to the reservoir temperature. This picture is complicated by the kinematics of collisions in the presence of the ion trap, the possibly slow relaxation of molecular internal states, unwanted chemistry, and a variety of 'experimental issues' that must be mitigated. In this section, each of these issues and its solution is described in turn.

\section{Collisional kinematics}

Since the early work of Major and Dehmelt [23] it has been known that, in apparent contradiction with the laws of thermodynamics, a gas of ions trapped in a radio-frequency (rf) Paul trap and immersed in a reservoir of neutral atoms does not equilibrate to the same temperature as the neutral atoms. Instead, the ions are found to have a higher temperature than the neutral gas, and in some cases are heated so much that they escape the trap. It was known that this phenomenon is due to the fact that ions are subject to a timedependent confining potential and are therefore not an isolated system. However, despite pioneering work by Dehmelt and others [24, 25], an accurate analytical description of the relaxation process was not achieved until very recently [26-29].

Building upon the important work of Moriwaki et al. [24], we have developed a kinematic model [27-29] over the last three years, which accurately describes the ion relaxation process in the presence of a buffer gas, as well as the heating effect that occurs when multiple ions are trapped simultaneously. This model, which has been verified by detailed molecular dynamics simulations $[27,28]$ and experiment when possible $[27,29]$, provides a simple and accurate means to calculate both the relaxation dynamics and the properties of the ion steady-state. Further, this model has been shown to agree with a quantum mechanical calculation of a single ion immersed in a buffer gas over its range of validity [30]. The most striking feature of the system, revealed and understood by the model, is a significant departure from equilibrium thermodynamics denoted 
by power law probability distributions and a bifurcation in the ion steady-state temperature when parameterized by trapped ion number. This treatment provides significant physical intuition for the problem and suggests several ways for optimizing ongoing and planned experiments. In the remainder of this section, the results and use of the model are described.

\section{Relaxation of a single ion in a buffer gas}

The equilibration of a test particle immersed in an ideal gas is a well known statistical physics problem, which is accurately realized in the technique of buffer-gas cooling of magnetically-trapped neutral particles with cryogenic helium [31]. While sympathetic cooling of charged particles in an ion trap shares many features with this 'traditional' buffer-gas cooling of neutral particles, the time dependence of the confining potential fundamentally alters the apparent energy conservation of the collision process in two unique ways.

First, the trajectory, $r_{j}$, and velocity, $v_{j}$, of an ion in a linear Paul trap, driven at $\mathrm{rf}$ frequency $\Omega$, can be expanded as a linear superposition of two orthogonal Mathieu functions $c\left(a_{j}, q_{j} ; \tau\right)$ and $s\left(a_{j}, q_{j} ; \tau\right)$ with coefficients $A_{j}$ and $B_{j}$,

$$
\begin{aligned}
& r_{j}(\tau)=A_{j} c_{j}(\tau)+B_{j} s_{j}(\tau) \\
& v_{j}(\tau)=A_{j} \dot{c}_{j}(\tau)+B_{j} \dot{s}_{j}(\tau)
\end{aligned}
$$

where $j=x, y, z, \tau=\Omega t / 2$, and the dependence on the Mathieu parameters $\left(\left\{a_{x}, a_{y}, a_{z}\right\}=\right.$ $\{-a,-a, 2 a\}$ and $\left\{q_{x}, q_{y}, q_{z}\right\}=\{q,-q, 0\}$ with $q=\frac{4 e V_{r f}}{m r_{0}^{2} \Omega^{2}}$ and $\left.a=\frac{4 \alpha e U_{e c}}{m z_{0}^{2} \Omega^{2}}\right)$ is suppressed [23] - here, $V_{r f}$ is the amplitude of the oscillating voltage assuming two of the four rods are held at ground, $m$ is the ion mass, and $r_{o}$ is the trap field radius. The Fourier transform of $c_{j}(\tau)$ and $s_{j}(\tau)[32]$ is a discrete spectrum,

$$
c_{j}(\tau)+\imath s_{j}(\tau)=\sum_{n=-\infty}^{\infty} C_{2 n} e^{l\left(\beta_{j}+2 n\right) \tau} .
$$

The $n=0$ term corresponds to the 'typical' motion of a harmonic oscillator, which occurs at frequency $\omega_{j}=\beta_{j} \Omega / 2$. $\beta_{j}$ can be approximated to sufficient accuracy for most purposes as [33]

$$
\beta_{j} \approx \sqrt{a_{j}-\frac{\left(a_{j}-1\right) q_{j}^{2}}{2\left(a_{j}-1\right)^{2}-q_{j}^{2}}-\frac{\left(5 a_{j}+7\right) q_{j}^{4}}{32\left(a_{j}-1\right)^{3}\left(a_{j}-4\right)}} .
$$

The remaining terms with $n \neq 0$ represent the components of the ion motion driven by the rf field - i.e. the so-called micromotion.

It is the presence of the $n \neq 0$ terms that is the critical difference between sympathetic cooling in static and time-dependent traps, and gives rise to the first type of heating effect, termed micromotion interruption. Consider the sinuousdial motion of a particle in a static trap, shown in Fig. 2a for one dimension of its motion. If a collision happens at position $x=a$ and $t=0$ that reduces the velocity such that $v_{x}^{\prime}=0$ after the collision, the trapped particle of mass $m$ begins a 'new' oscillation trajectory $x^{\prime}=a \cos (2 \pi \sqrt{k / m} t)$, where $k$ is the trap spring constant. This collision always reduces the total energy of the particle. By contrast, in the time-dependent potential of a linear Paul trap, because of the 

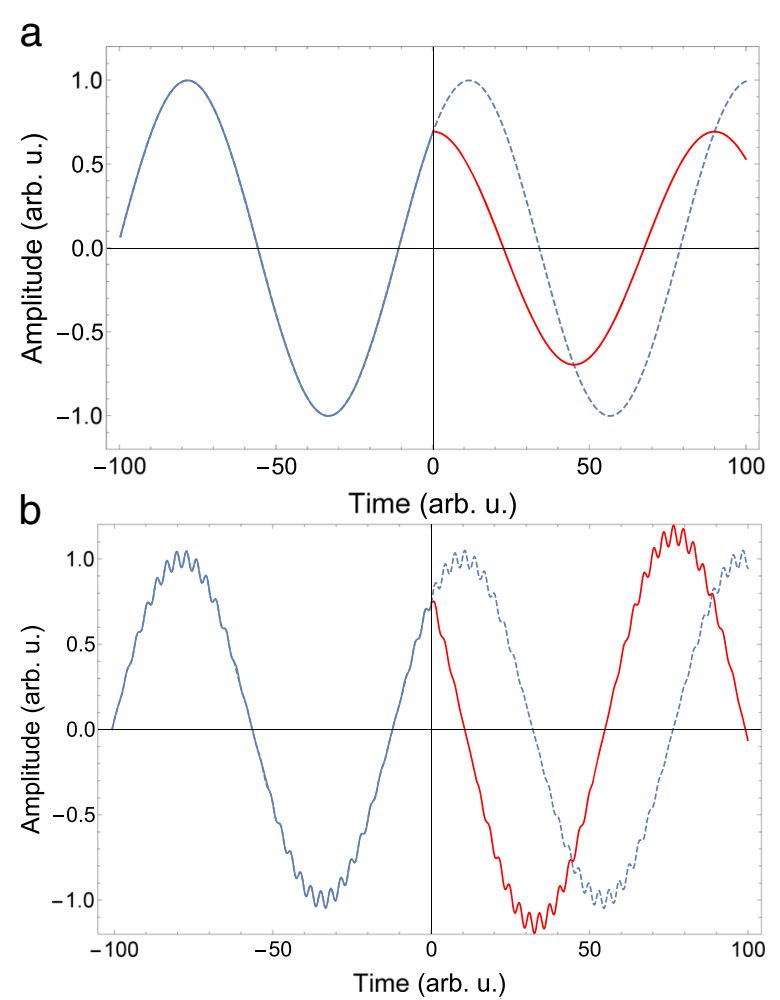

Fig. 2 Sample trajectories showing the effect of a collision at $t=0$ that reduces the trapped particle velocity to zero in a $\mathbf{a}$ static and $\mathbf{b}$ if Paul trap. In the static case, a stopping collision always leads to cooling (i.e. reduced amplitude of the oscillation), however, in the time-dependent potential of the Paul trap a stopping collision can lead to heating

terms in Eq. 2 with $n \neq 0$, it is possible that even though the collision brings the particle to rest, the particle may have a higher energy after the collision. This can be seen by again considering a collision that leads to $v_{x}^{\prime}=0$, which, depending on the rf phase and position of the ion, could be accomplished by having large and opposite contributions to the velocity from the $n=0$ (secular) mode and $n \neq 0$ (micromotion) modes. Thus, even though the particle is momentarily stopped, it could leave the collision on a trajectory of higher amplitude - a sample such trajectory is shown in Fig. 2b. Mathematically, this phenomenon occurs because one is not free to choose the $C_{2 n}$ in Eq. 2, but must find them from the Mathieu recursion relation [33] for the scaled time, i.e. the rf phase, of the collision. Physically, this heating occurs because the collision may perturb the ion trajectory such that its position and velocity after the collision are such that they belong to a higher energy trajectory. It is for these reasons, we refer to this type of heating as micromotion interruption heating.

The second type of heating, first understood in [26], is closely related, but occurs due to the finite time of the actual collision event; here the 'time' required for the collision is loosely defined as the duration of time that the atom-ion interaction is significant compared to the trapping force. When a collision occurs with the neutral buffer gas the trajectory of the ion is primarily given by the collision dynamics, with the rf field of the trap playing the role of a perturbation. Depending on the position of the ion when the collision begins, the phase of the rf field, and the trajectory during the collision, the $\mathrm{rf}$ 
field can do net work on the ion during the collision; sometimes this work removes energy from the motion of the ion and at other times it adds energy. As this heating occurs during the actual collision it sets the limit on the minimum attainable temperature and makes it difficult (but not impossible) to observe single partial wave collisions in a MOTion trap [26]. In most practical situations micromotion interruption heating is much larger than this second type of heating and therefore we do not consider it further; however, a very recent experiment has reported observation of this limit [34].

Several useful points follow immediately from the analysis of micromotion interruption heating. First, the steady-state average temperature $T_{j}$ (here temperature is an approximate term and indicates the average energy per $k_{B}$ ) of an ion immersed in a buffer gas of average temperature $T_{n}$ is given as [27]:

$$
\begin{aligned}
\frac{T_{x}}{T_{n}}=\frac{T_{y}}{T_{n}} & =\frac{9(2+\tilde{m}) \alpha}{18-3 \tilde{m}(\alpha+4 \epsilon-4)-2 \tilde{m}^{2}(\alpha+2 \epsilon-1)} \\
\frac{T_{z}}{T_{n}} & =\frac{3[6+\tilde{m}(2+\alpha-4 \epsilon)]}{18-3 \tilde{m}(\alpha+4 \epsilon-4)-2 \tilde{m}^{2}(\alpha+2 \epsilon-1)}
\end{aligned}
$$

where $\alpha \approx 2+2 q^{2.24}, \epsilon \approx 1+2.4 q^{2.4}$, and $\tilde{m}$ is the ratio of the neutral mass to the ion mass, $m_{\mathrm{n}} / m_{\mathrm{i}}$. The ion relaxes from its initial average temperature towards this steadystate value at a rate:

$$
\dot{Q}_{i-n}=-\Gamma_{L} \lambda(T-\bar{T}) .
$$

$\Gamma_{L}=2 \pi \rho \sqrt{\frac{\alpha}{2 \mu}}$ is the Langevin collision rate, where $\rho$ is the neutral gas density, $\alpha$ is the neutral atom polarizability, and $\mu$ is the collision complex reduced mass. $\bar{T}$ is the average of the temperatures in Eq. 4. The kinetics factor, which is the smallest eigenvalue of a three-dimensional relaxation matrix [28], is given as

$$
\lambda=\frac{\tilde{m}}{(1+\tilde{m})^{2}}\left(1-\frac{\tilde{m}}{\tilde{m}_{c}}\right)
$$

The term in parentheses in Eq. 6 constitutes the main departure from the sympathetic cooling rate in a static trap. The critical mass ratio is:

$$
\tilde{m}_{c}=\frac{3\left(4-\alpha-4 \epsilon+\sqrt{\alpha^{2}+8 \alpha(1+\epsilon)+16 \epsilon^{2}}\right)}{4(2 \epsilon+\alpha-1)} .
$$

For $\tilde{m} \leq \tilde{m}_{c}$, the ion is sympathetically cooled to $T_{j}$ at the given rate; however, once $\tilde{m}$ exceeds $\tilde{m}_{c}$ the ion is heated by the neutral gas, regardless of the gas temperature, until it is lost from the trap.

The impact of Eqs. 4-7 is illustrated in Fig. 3. Here, the normalized cooling rate, $\lambda$, is plotted as a function of $m_{i}$ and $m_{n}$ for $q=0.2$. The maximum cooling rate is achieved for $\tilde{m}=2 /\left(2+\tilde{m}_{c}\right)$ or, in this case, $m_{\mathrm{n}} \approx 0.38 m_{\mathrm{i}}$. Overlaid on this graph are lines of constant $T_{\mathrm{x}} / T_{\mathrm{n}}$, which are labeled by the value of the ratio. For example, sympathetic cooling of an ion with mass $\sim 100 \mathrm{amu}$ is most efficient with a neutral mass of $\sim 40 \mathrm{amu}$ and leads to an ion with a steady-state energy roughly $3 \times$ that of the neutral atoms. Also shown in the graph as individual points are the various hybrid atom-ions systems that have been realized thus far. While some of the systems do lie in the unstable region, those experiments are able to operate by using some combination of a low buffer gas density, laser cooling of trapped ions, and a multipole trap [35]. 


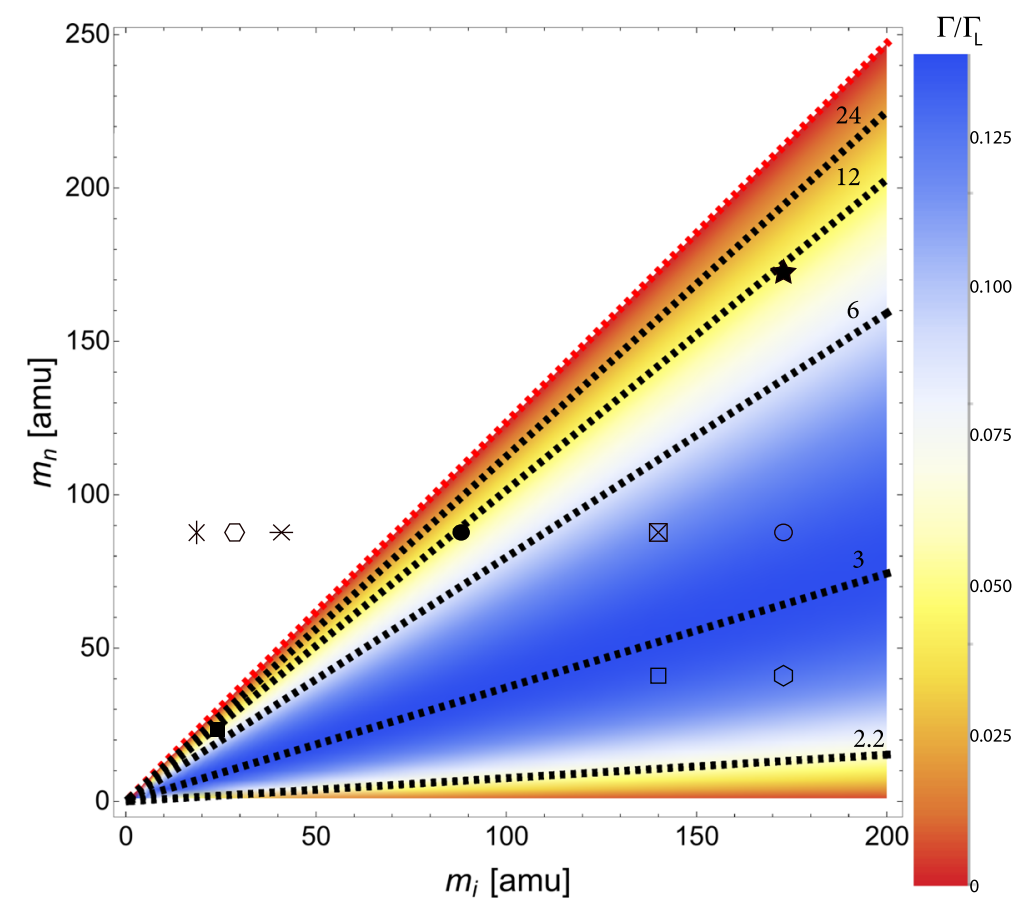

Fig. 3 Normalized cooling rate contour plot as a function of ion and neutral masses for $q=0.2$. Lines of constant $T_{\mathrm{x}} / T_{\mathrm{n}}$, labeled by the value of the ratio, are shown as (thick dashed) black lines. Plot markers indicate experimentally realized systems: $\star \mathrm{Yb} \mathrm{Yb}^{+}, \mathrm{MIT}[72] ; \bigcirc \mathrm{Rb}-\mathrm{Yb}^{+}$, Bonn [73] and Ulm [74]; $\otimes \mathrm{Rb}^{+} \mathrm{Ba}^{+}, \mathrm{UIm}[74]$ and Basel [75]; $\bigcirc \mathrm{Ca}^{-} \mathrm{Yb}^{+}, \mathrm{UCLA}[76]$ and $\mathrm{Ca}-\mathrm{BaCl}{ }^{+}, \mathrm{UCLA}[41] ; * \mathrm{Rb}-\mathrm{Ca}{ }^{+}$, Basel [44]; $\square \mathrm{Ca}-\mathrm{Ba}^{+}, \mathrm{UCLA}_{\text {[45]; }}$ $\mathrm{Na}-\mathrm{Na}^{+} \mathrm{UCONN}[77] ; * \mathrm{Rb}^{-\mathrm{OH}^{-}}$, Innsbruck [78]; $\bigcirc \mathrm{Rb}-\mathrm{N}_{2}^{+}$, Basel [18]; $\mathrm{Rb}-\mathrm{Rb}^{+}$Bangalore [79] and $\mathrm{Rb}^{-\mathrm{Sr}^{+}}$, Weizmann [34]

A further peculiarity of sympathetic cooling in an ion trap is manifested in the steadystate energy distribution of the ion, which features a power-law tail, of the form $P(E) \propto$ $E^{-(v+1)}$, due to the random amplification of the ion energy by collisions; it is for this reason that temperature is an ill-defined quantity for the ion and we instead refer to the steady-state (average) energy in the above. In [28] a simplified model is considered to gain a qualitative understanding of how this distribution arises. If the motion of the ion and neutral atom are restricted to one dimension, it can be shown that the effect of collision is to cause in a random walk in phase space which has both additive and multiplicative terms. As is well known in statistics, multiplicative terms in a random walk give rise to a power-law distribution. Requiring that the steady-state distribution does not change in time, it is possible to estimate that the power law tail of the energy probability density goes as $P(E) \propto E^{-(v+1)}$ with $v \approx 3.3 / \tilde{m}-1.3$, which agrees well with molecular dynamics simulations and has been confirmed in a very recent experiment [34].

\section{Sympathetic cooling of multiple ions}

The previous treatment is exclusively for a single ion immersed in a neutral buffer gas. Once multiple ions are held in the ion trap, the ions no longer experience pure Mathieu trajectories and a variety of new effects are possible. In the case of sympathetic cooling the most important new phenomenon is ion-ion heating, which occurs because as the ions perturb the trajectories of co-trapped ions it is possible for the rf trap to do net work 
on them. With a treatment similar to the above we were able to show that this effect represents a heating rate for the trapped ion sample of [27]:

$$
\dot{Q}_{i-i}=\frac{e^{4} \rho \ln \Lambda}{2 \pi \epsilon_{o}^{2} \sqrt{m_{i}}\left(3 k_{B} \eta T\right)^{3 / 2}} \bar{\epsilon} T \xi\left(N_{i}\right)
$$

where $T$ is the average total energy of the ions per $k_{B}, \eta$ is the ratio of the total energy to secular energy ( $\eta \sim 1 / 2-3 / 5$ for typical ion trap parameters); $\rho$ is the density of the trapped ion sample; $\xi\left(N_{i}\right)=1-e^{-\left(N_{i} / N_{k}\right)^{3}}$ where $N_{i}$ is equal to the number of trapped ions and $N_{k}$ is the trapped ion number at which a cooled linear crystal of ions 'kinks'; and

$$
\ln \Lambda= \begin{cases}f_{\mathrm{I}}(g)=\frac{\ln (1+0.7 / g)}{1+125 \sqrt{g}} & : g<1 \\ f_{\mathrm{II}}(g)=\frac{f_{\mathrm{I}}(g=1)}{g^{2}}\left(1+10 e^{-\eta T / T_{s}}\right) & : g \geq 1\end{cases}
$$

where the strong-coupling parameter $g=\sqrt{\rho} e^{3} /\left(4 \pi\left(\epsilon_{o} k_{B} T\right)^{3 / 2}\right)$ and $T_{s}$ is the temperature of ion crystallization. This expression for $\ln \Lambda$, first presented in [29] and shown in Fig. 4 extends the previous determination in [28] to the lower temperatures and trapped ion number of one-dimensional ion chains. Physically, $\ln \Lambda$, known as the Coulomb logarithm, accounts for ion many-body effects such as repulsion and shielding within the one component plasma.

Interestingly, the heating rate, $\dot{Q}_{i-i}$, goes towards zero at both high and low temperature. In the solid phase (low $T$ ), the ion motion is correlated - i.e. $\ln \Lambda$ becomes small and therefore very little energy is coupled into the ion motion from the trapping fields. In the gas phase (high $T$ ), though the ion motion is uncorrelated - i.e. $\ln \Lambda$ is large - the ion density is low and the ions are relatively non-interacting. Only in the liquid phase (intermediate $T$, typically $1-10 \mathrm{~K}$ ) are the ions both strongly interacting and uncorrelated, and thus it is in this regime where $\dot{Q}_{i-i}$ exhibits its maximum.

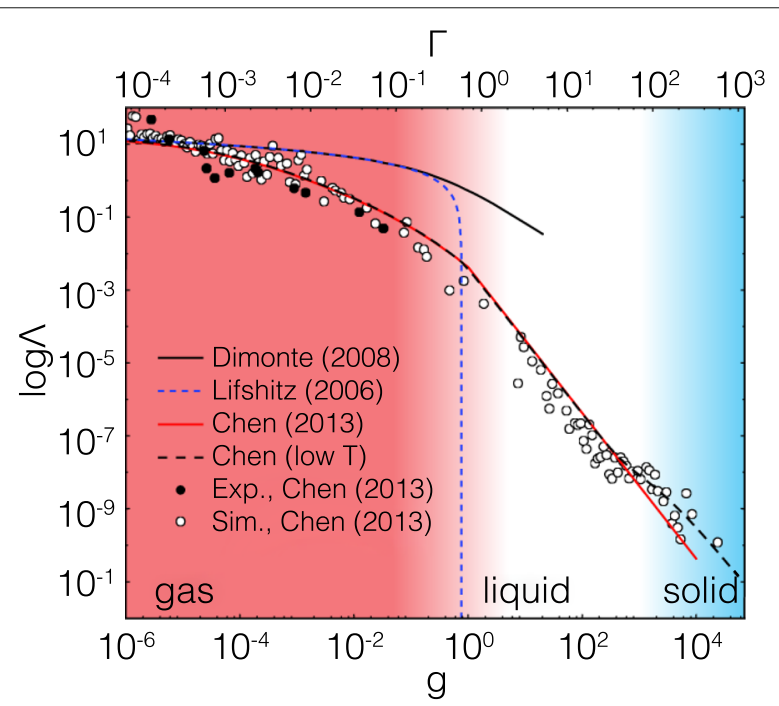

Fig. 4 The experimental and MD determinations of $\ln \Lambda$ versus $g$. Despite large variation in trap parameters the observed values fall along the same curve, indicating a universal form for $\ln \Lambda$. The dashed black curve represents the analytical form used here and in [29] (modified to agree with low temperature data), while the other curves represent previous forms described elsewhere [27]. The top $x$-axis denotes the domain of this graph in terms of the plasma coupling parameter, $\Gamma$, which is the ratio of potential to kinetic energy 
This behavior creates very rich dynamics for the cooling of ions, either by a laser or by a buffer gas. As the ions are cooled, the heating rate actually increases and whether or not the trapped sample can be cooled passed the liquid phase depends on whether or not the cooling rate can overwhelm this increasing heating rate [36]. By combining these treatments of heating and cooling in a hybrid trap, i.e. $\dot{Q}_{i-n}$ and $\dot{Q}_{i-i}$, we arrive at an analytical model that describes the temperature of multiple trapped ions immersed in a buffer gas. Qualitatively, three regimes exist and are shown in Fig. 5. Regime I, which is characterized by small ion number, features only one temperature, $T_{c}$, for which the heating (blue curve) and cooling (green curve) rates are balanced, implying the existence of a unique steady-state for that ion number. In regime II, a larger ion number shifts the heating curve upwards, creating two additional intersections between the heating and cooling rates at $T_{u}$ and $T_{h}$. Ions initialized with a temperature greater (less) than the unstable fixed point at temperature $T_{u}$ will evolve to the steady-state temperature $T_{h}\left(T_{c}\right)$. In regime III, an overabundance of ions causes the heating rate to overwhelm the cooling rate leaving only one intersection and steady-state temperature $T_{h}$. The boundaries of these regimes are marked by blue-sky, or saddle-node, bifurcations [37], where a second steady-state temperature is either created (I-II) or annihilated (II-III) simply by increasing the number of trapped ions by one.

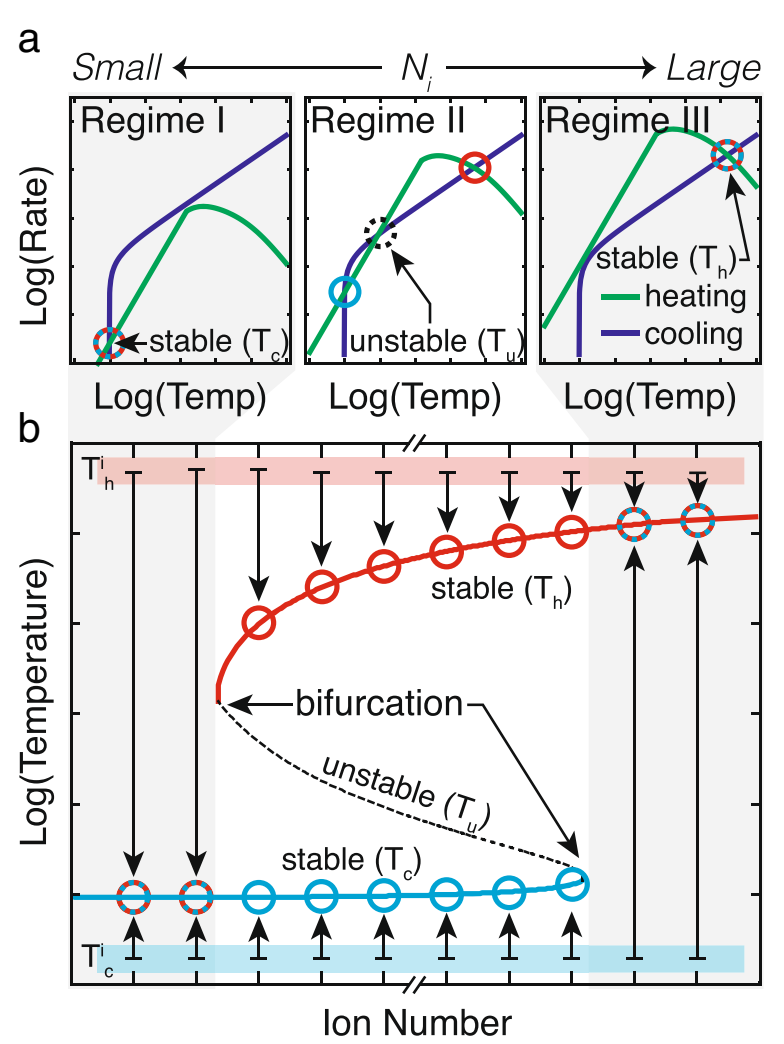

Fig. 5 Emergence of nonequilbrium dynamics in a hybrid atom-ion trap. a, Analytical heating and cooling rates for ions in a hybrid atom-ion trap. Three characteristic Regimes exist for different relative heating and cooling strengths as parameterized by $N_{i}$ and are characterized by the number and type of fixed points they exhibit. $\mathbf{b}$, A qualitative description of the bifurcations as transitions between the three Regimes. In Regime II, the steady-state ion temperature is dependent upon the initial temperature, whereas only one steady-state temperature exists for the other Regimes 
This bifurcation was recently experimentally observed [29], but its effects have been felt since the work of Dehmelt. For sympathetic cooling experiments the most practical implication is that it is very difficult to cool a large number of ions $\left(N_{i}>30\right)$ to the solid phase using only buffer gas cooling. Table 1 shows the temperatures possible using different buffer gas sources. For example, using a $4 \mathrm{~K}^{4} \mathrm{He}$ buffer gas with a density of $10^{14} \mathrm{~cm}^{-3}$, we estimate the steady-state ion temperature for $N_{i}\left(\mathrm{Ba}^{+}\right)>30$ to be $\sim 10 \mathrm{~K}$. In fact, using a $170 \mathrm{mK}^{3} \mathrm{He}$ buffer gas with the same density, we still estimate the steady-state ion temperature to be $\sim 10 \mathrm{~K}$. This illustrates the surprising diminishing returns of naively decreasing the buffer gas temperature to reach colder steady-state ion temperatures. To overcome this limitation, high-density buffer gases composed of lasercooled, highly-polarizable atoms, such as those created in hybrid traps, must be used (Table 1). However, even with current hybrid traps, for more than a critical number of ions, it is only possible to maintain, not create, steady-state ion temperatures near that of the laser-cooled buffer gas. For still larger ion numbers and with certain buffer gases (typical low-density alkaline-earth gases), it may not even be possible to maintain these laser-cooled temperatures (for example, with $\mathrm{Ca}$ ). In most cases, these issues can be circumvented either by directly laser-cooling the trapped ions or by sympathetically cooling the ions with co-trapped laser-cooled ions, which can maintain (or simply initialize, in the case of cooling with $\mathrm{Na}$ and $\mathrm{Rb}$ gases) ions at sub-millikelvin temperatures.

\section{Summary and guidelines for efficient sympathetic cooling}

In summary, we have developed an analytical model, bolstered by detailed molecular dynamics simulations and experimental data, that accurately predicts the steady-state value and dynamics of the energy of a single ion immersed in a neutral buffer gas. The transition from sympathetic cooling to heating, and its dependence on trap parameters and masses of the particles have also been explained. Recent excellent work has extended this treatment from quadrupole traps to multipole ion traps as well as explored the dependence on buffer gas size [35]. In addition, we have confirmed that the recent observation of non-Maxwellian statistics [17] for a trapped ion can be attributed to random heating collisions and provided a means to approximate the expected power law of the energy distribution. These results solve the longstanding issues and questions that have existed since Dehmelt first considered this problem over forty years ago.

We have also developed an analytical model, which accurately explains the phenomenon of ion-ion heating through the use of the Coulomb logarithm parameter from plasma physics. Taken together these two models provide an approximate analytical solution to the most general situation of multiple ions immersed in a buffer gas.

Table 1 Expected steady-state temperatures of $\mathrm{Ba}^{+}$ions for the large $\mathrm{N}_{i}$ limit after being immersed in a variety of gases with polarizability $\alpha$, density $\rho$, and temperature $T_{n}$

\begin{tabular}{lllllll}
\hline Gas & $\alpha$ (a.u. $)$ & $\rho\left(\mathrm{cm}^{-3}\right)$ & $T_{n}$ & $T_{\text {create }}$ & $T_{u}$ & $T_{\text {maintain }}$ \\
\hline${ }^{3} \mathrm{He}$ & 1.38 & $10^{14}$ & $170 \mathrm{mK}$ & $10 \mathrm{~K}$ & $2 \mathrm{~K}$ & $300 \mathrm{mK}$ \\
${ }^{4} \mathrm{He}$ & 1.38 & $10^{14}$ & $4 \mathrm{~K}$ & $10 \mathrm{~K}$ & - & - \\
${ }^{23} \mathrm{Na}$ & 163 & $10^{12}$ & $100 \mu \mathrm{K}$ & $30 \mathrm{~K}$ & $800 \mathrm{mK}$ & $200 \mu \mathrm{K}$ \\
${ }^{87} \mathrm{Rb}$ & 319 & $10^{12}$ & $10 \mu \mathrm{K}$ & $30 \mathrm{~K}$ & $800 \mathrm{mK}$ & $40 \mu \mathrm{K}$ \\
${ }^{40} \mathrm{Ca}$ & 157 & $10^{10}$ & $4 \mathrm{mK}$ & $300 \mathrm{~K}$ & - & - \\
\hline$T_{\text {create }}\left(T_{\text {maintain }}\right)$ & is the temperature reached if the ions are initialized are &
\end{tabular}

$T_{\text {create }}\left(T_{\text {maintain }}\right)$ is the temperature reached if the ions are initialized above (below) $T_{u}$ 
With this analytical solution it is now possible to establish guidelines for efficient sympathetic cooling in a MOTion trap. To cool an ion of mass $m_{\mathrm{i}}$ a buffer gas atom of mass $m_{\mathrm{n}} \leq m_{\mathrm{i}} / 2$ should be used. The ion trap should be operated with the smallest number of ions and the lowest Mathieu $q$ experimentally possible. Significant departure from these guidelines, e.g. trapping a large number of ions, is possible if a sample of lasercooled atomic ions are co-trapped with the ion of interest. Then sympathetic cooling by the laser-cooled ions can easily overcome these effects. Typically, our experiments run in this mode, where laser-cooled atomic ions provide sympathetic cooling of the molecular ion motion and the neutral buffer gas provides cooling of the molecular internal states as described in the next section.

\section{Caveats and assumptions of this treatment}

In the development of the above model of sympathetic cooling several assumptions have been made. These assumptions include a uniform neutral atom density so that the collision rate does not depend on position; a differential scattering cross-section as predicted by a long-range potential of the form $-C_{4} / r^{4}$; the lack of excess micromotion; and the lack of reactive collisions and production of unwanted ions. Some of these assumptions are relaxed in the original treatment, e.g. the lack of excess micromotion [27]; nonetheless, deviations from these assumptions can become important. In those cases, detailed molecular dynamics simulations like those performed in [25, 29, 35, 36, 38, 39] are necessary.

\section{Cooling of molecular ion rotational and vibrational motion}

Removal of energy stored in long-lived vibrational levels is particularly problematic because optical transitions between vibrational levels are not governed by strict selection rules, making laser cooling difficult. Additionally, sympathetic cooling methods are often inefficient at quenching molecular vibrational motion. For example, in buffer-gas cooling of neutral molecules by cryogenic helium it is observed that while the molecular rotational degree of freedom quickly equilibrates to the helium temperature, the molecular vibration does not. In fact, it is estimated that between $10^{6}$ to $10^{8}$ collisions are required before the molecular vibration is relaxed by one vibrational quanta [40].

We argued in 2009 [13] that the details of the ion-neutral interaction would result in near-unit efficiency for vibrational relaxation, and in 2013 showed that for the case of $\mathrm{Ca}+\mathrm{BaCl}^{+}$approximately one in five collisions led to vibrational quenching [41]. A full quantum mechanical treatment of this relaxation has recently been completed and shows good agreement with the measured rate constant [42]. These calculations show that the efficient relaxation is due to the fact that the strong, long-range atom-ion interaction significantly perturbs the molecular bound length. This couples the vibrational energy to the center of mass energy of the collision complex, allowing for efficient vibrational relaxation.

While each specific atom-ion case technically requires a full quantum scattering calculation, [42] establishes a statistical capture model which can be used to estimate the relative vibrational quenching efficiency. In this model, the quenching rate is proportional to the scattering rate, given by the Langevin rate constant, and the probability of quenching, which is proportional to the number of accessible vibrational channels in the three-body complex. Estimating the latter term as the dissociation energy of the triatomic 
ion, $D$, divided by the vibrational frequency of the diatomic ion, $\omega$, the quenching rate goes as $k \propto \frac{D}{\omega} \sqrt{\frac{\alpha}{\mu}}$. With this expression, it is now possible to establish general guidelines for efficient vibrational cooling. First, the neutral atom should have as large a static polarizability as possible; practically speaking the polarizability of all laser-coolable atoms is sufficient. Second, the molecular ion should have a large permanent dipole moment. This ensures that the atom-ion interaction is anisotropic enough to efficiently couple the neutral atom motion to the molecular ion bond. Further, molecular ions with a smaller vibrational frequency are preferred, though if the triatomic complex is deeply bound this requirement can be relaxed. In total, these guidelines are easily satisfied for a large number of interesting atom-molecular ion combinations.

Finally, though rotational quenching of a molecular ion by an ultracold buffer gas has not yet been measured, it is expected to be quite efficient. This expectation is based on both theoretical work [42] and rotational quenching measurements using cryogenic $\mathrm{He}$ buffer gases [19], which find quenching rates on the order of one-tenth of the Langevin rate. Therefore as long as the aforementioned requirements are satisfied, sympathetic cooling of the internal degrees of freedom of molecular ions in the MOTion trap appears quite general.

\section{Unwanted chemistry}

For efficient sympathetic cooling, the molecular ion must be able to collide with the neutral atom coolant without charge exchange or chemical reactions taking place. Since an ion can lower its energy by accepting an electron, it is necessary to use an atomic buffer gas with a high ionization potential. Tables 2 and 3 show the ionization potentials of candidate buffer gas atoms and the electron affinities of a sample of molecular ions, respectively. The first test for compatibility is that the ion electron affinity must be less than the atom ionization potential to prevent charge exchange reactions; for example, in the case of a Ca buffer gas with an ionization potential of $6.2 \mathrm{eV}$, all the ions with electron affinity less than $\mathrm{UC}^{+}$in the table will not charge exchange with ground-state $\mathrm{Ca}$. In the case of $\mathrm{Rb}$ buffer gas, none of the ions with electron affinity less than $\mathrm{KNa}^{+}$will undergo ground-state charge exchange.

Once ground-state charge exchange reactions are precluded there are a variety of other reactions that must be considered on a case-by-case basis to determine if a chosen atomion pair is compatible. For illustration, in the case of $\mathrm{Ca}$ and $\mathrm{BaCl}^{+}$these are:

$$
\begin{array}{cl}
\text { cation substitution : } & \mathrm{Ca}+\mathrm{BaCl}^{+} \longrightarrow \mathrm{Ba}+\mathrm{CaCl}^{+} \\
\text {cation replacement : } & \mathrm{Ca}+\mathrm{BaCl}^{+} \longrightarrow \mathrm{Ba}^{+}+\mathrm{CaCl} \\
\text { anion substitution : } & \mathrm{Ca}+\mathrm{BaCl}^{+} \longrightarrow \mathrm{Cl}+\mathrm{BaCa}^{+} \\
\text {anion replacement : } & \mathrm{Ca}+\mathrm{BaCl}^{+} \longrightarrow \mathrm{Cl}^{+}+\mathrm{BaCa}^{-} \\
\text {double charge transfer : } & \mathrm{Ca}+\mathrm{BaCl}^{+} \longrightarrow \mathrm{Ca}^{-}+\mathrm{BaCl}^{2+} \\
\text { radiative association : } & \mathrm{Ca}+\mathrm{BaCl}^{+} \longrightarrow \mathrm{CaBaCl}^{+}+\gamma
\end{array}
$$

Calculating whether these reactions are energetically possible requires knowledge of the dissociation energy, electron affinity, and ionization energy of the ground states of each of the atoms and molecules involved. In the case of the $\mathrm{Ca}+\mathrm{BaCl}^{+}$system explored in [41] all relevant information is available and all channels, except for radiative association, are energetically precluded for the ground state. Unfortunately, these reaction energetics 
Table 2 Properties of potential ultracold atomic buffer gases

\begin{tabular}{lllll}
\hline Atom & $\mathrm{IP}[\mathrm{eV}]$ & $\lambda[\mathrm{nm}]$ & $\mathrm{IP} /(\mathrm{hc} / \lambda)$ & $\mathrm{m}$ [amu] \\
\hline $\mathrm{Hg}$ & 10.437504 & 254 & 2.14 & 201 \\
$\mathrm{Zn}$ & 9.3941990 & 214 & 1.62 & 65 \\
$\mathrm{Be}$ & 9.322699 & 235 & 1.77 & 9 \\
$\mathrm{Cd}$ & 8.993822 & 229 & 1.66 & 112 \\
$\mathrm{Mg}$ & 7.646235 & 285 & 1.76 & 52 \\
$\mathrm{Cr}$ & 6.76651 & 425 & 2.32 & 173 \\
$\mathrm{Yb}$ & 6.254159 & 399 & 2.01 & 169 \\
$\mathrm{Tm}$ & 6.18431 & 410 & 2.04 & 40 \\
$\mathrm{Ca}$ & 423 & 2.09 & 167 \\
$\mathrm{Er}$ & 6.11315520 & 583 & 2.87 & 165 \\
$\mathrm{Ho}$ & 6.1077 & 410 & 1.99 & 162 \\
$\mathrm{Dy}$ & 6.0215 & 421 & 2.02 & 88 \\
$\mathrm{Sr}$ & 561 & 2.12 & 7 \\
$\mathrm{Li}$ & 5.93905 & 671 & 2.92 & 137 \\
$\mathrm{Ba}$ & 5.69486720 & 554 & 2.33 & 23 \\
$\mathrm{Na}$ & 5.391714761 & 589 & 2.44 & 39 \\
$\mathrm{~K}$ & 5.211664 & 767 & 2.69 & 85 \\
$\mathrm{Rb}$ & 5.1390767 & 780 & 2.63 & \\
\hline
\end{tabular}

The second column shows the energy required to ionize the atom, which is a indicative of its reactivity with candidate ions. The third column shows the lowest wavelength required for laser cooling of the atom. The shorter the wavelength the more potential there is for problem such as unwanted ionization, dissociation, or production of photoelectrons. The fourth column is the approximate number of laser cooling photons required to reach the ionization energy of the atom. The fifth column shows the mass of the atom for reference. Roughly speaking, efficient cooling is only possible for ions with a mass roughly two to three times this mass

Table 3 Partial list of the electronic affinities of candidate molecular ions

\begin{tabular}{|c|c|c|c|c|c|c|c|c|c|}
\hline Molecule & $\mathrm{EA}[\mathrm{eV}]$ & Molecule & $\mathrm{EA}[\mathrm{eV}]$ & Molecule & $\mathrm{EA}[\mathrm{eV}]$ & Molecule & $\mathrm{EA}[\mathrm{eV}]$ & Molecule & $\mathrm{EA}[\mathrm{eV}]$ \\
\hline$\overline{\mathrm{Rb}_{2}^{+}}$ & 3.5 & $\mathrm{Li}_{2}^{+}$ & 5.1 & $\mathrm{YH}_{2}^{+}$ & 6.2 & $\mathrm{TiS}^{+}$ & 7.1 & $\mathrm{Nal}^{+}$ & 7.64 \\
\hline $\mathrm{Cs}_{2}^{+}$ & 3.6 & $\mathrm{SrF}^{+}$ & 5.3 & $\mathrm{HoF}^{+}$ & 6.2 & $\mathrm{GeCl}^{+}$ & 7.2 & $\mathrm{RbBr}^{+}$ & 7.7 \\
\hline $\mathrm{K}_{2}^{+}$ & 4.1 & $\mathrm{SrCl}^{+}$ & 5.5 & $\mathrm{Ti}_{2}^{+}$ & 6.3 & $\mathrm{Csl}^{+}$ & 7.25 & $\mathrm{Pd}_{2}^{+}$ & 7.7 \\
\hline $\mathrm{KNa}^{+}$ & 4.4 & $\mathrm{SrBr}^{+}$ & 5.5 & US $^{+}$ & 6.3 & $\mathrm{SiF}^{+}$ & 7.26 & $\mathrm{C}_{6} \mathrm{H}_{7} \mathrm{~N}^{+}$ & 7.7 \\
\hline $\mathrm{KLi}^{+}$ & 4.6 & $\mathrm{Srl}^{+}$ & 5.5 & $\mathrm{ErF}^{+}$ & 6.3 & $\mathrm{GeBr}^{+}$ & 7.3 & (Aniline) & \\
\hline $\mathrm{Bal}^{+}$ & 4.7 & $\mathrm{CaBr}^{+}$ & 5.6 & $\mathrm{TiO}^{+}$ & 6.4 & $\mathrm{SnF}^{+}$ & 7.4 & $\mathrm{LiD}^{+}$ & 7.7 \\
\hline $\mathrm{BaBr}^{+}$ & 4.8 & $\mathrm{UO}^{+}$ & 5.7 & $V_{2}^{+}$ & 6.4 & $\mathrm{SnBr}{ }^{+}$ & 7.4 & $\mathrm{CsBr}^{+}$ & 7.72 \\
\hline $\mathrm{SrF}^{+}$ & 4.9 & $\mathrm{CaF}^{+}$ & 5.8 & $\mathrm{CaO}^{+}$ & 6.5 & $\mathrm{Mn}_{2}^{+}$ & 7.4 & $\mathrm{PbBr}^{+}$ & 7.8 \\
\hline $\mathrm{CeO}^{+}$ & 4.9 & $\mathrm{CaH}^{+}$ & 5.9 & $\mathrm{SnCl}+$ & 6.6 & $\mathrm{Si}_{2}^{+}$ & 7.4 & $\mathrm{BSi}^{+}$ & 7.8 \\
\hline $\mathrm{BaF}^{+}$ & 4.9 & $\mathrm{CaCl}^{+}$ & 6 & $\mathrm{TiO}^{+}$ & 6.8 & $\mathrm{MgCl}^{+}$ & 7.5 & $\mathrm{MgF}^{+}$ & 7.8 \\
\hline $\mathrm{PrO}^{+}$ & 4.9 & DyF $^{+}$ & 6 & $\mathrm{BaO}^{+}$ & 6.9 & $\mathrm{PbF}^{+}$ & 7.5 & $\mathrm{~N}\left(\mathrm{CH}_{3}\right)_{3}^{+}$ & 7.82 \\
\hline $\mathrm{Na}_{2}^{+}$ & 4.9 & $\mathrm{TaO}^{+}$ & 6 & $\mathrm{UN}^{+}$ & 7 & $\mathrm{PbCl}^{+}$ & 7.5 & (Trimethylamine ) & \\
\hline $\mathrm{LaO}^{+}$ & 4.9 & $\mathrm{TiH}^{+}$ & 6 & $\ln \mathrm{S}^{+}$ & 7 & $\mathrm{~N}\left(\mathrm{CH}_{2} \mathrm{CH}_{3}\right)_{3}^{+}$ & 7.5 & $\mathrm{LiH}^{+}$ & 7.85 \\
\hline $\mathrm{BaCl}^{+}$ & 5.0 & $\mathrm{SrO}^{+}$ & 6.1 & $\mathrm{ZrH}_{2}^{+}$ & 7 & (Triethylamine) & & $\mathrm{Cu}_{2}^{+}$ & 7.89 \\
\hline $\mathrm{LiNa}^{+}$ & 5.0 & $\mathrm{ZrO}^{+}$ & 6.1 & $\operatorname{lnS} e^{+}$ & 7.1 & $\mathrm{HfO}^{+}$ & 7.5 & $\mathrm{PtSi}^{+}$ & 7.9 \\
\hline $\mathrm{VO}^{+}$ & 5.0 & $\mathrm{Cal}^{+}$ & 6.1 & $\mathrm{TiS}^{+}$ & 7.1 & $\mathrm{GeF}^{+}$ & 7.5 & $\mathrm{Ge}_{2}{ }^{+}$ & 7.9 \\
\hline $\mathrm{NdO}^{+}$ & 5.0 & $\mathrm{UC}^{+}$ & 6.2 & $\mathrm{Rb}^{+}$ & 7.1 & $\operatorname{lnTe} e^{+}$ & 7.6 & $\mathrm{ZrN}^{+}$ & 7.9 \\
\hline
\end{tabular}

Charge exchange is forbidden in collisions with a neutral atom if the molecule EA is smaller than the IP of the neutral atom. We emphasize that this is only a partial list of molecules and that the number of molecular ions that can be cooled by this technique is likely much larger. Data taken from [80-82] 
are not known for many molecular ions as the the dissociation energy is often difficult to find in the literature. Nonetheless, for the lighter diatomic molecular ions commercial quantum chemistry packages should provide these values with sufficient accuracy to determine if the reactions proceed or are energetically precluded. Further, sometimes reactions can be excluded based on general arguments. For example, for most potential buffer gas atoms and ionically bonded molecular ions, anion substitution and replacement reactions are unlikely. Likewise, the radiative association channel is generically always allowed, however, the reaction rate will typically be $\leq 10^{-14} \mathrm{~cm}^{-3}[43]$ and therefore much slower than other experimental timescales.

While many atom-molecular ion combinations can be found where ground state reactions are energetically precluded, it is very difficult, if not impossible, to find combinations where excited-state reactions are also precluded. This would be appear to be problematic since the operation of a MOT requires a large fraction of excited atoms. Fortunately, nature is sometimes kind. Because the polarizability of the ground state and excited state of an atom are typically very different, as the ion approaches the atom the levels are Stark shifted by very different amounts. This causes the lasers used for cooling to no longer be resonant with the transition and the excitation process is halted. Therefore, it is only possible to excite an atom when it is far from the ion, typically a few $100 a_{0}$. And since the atom and ion are moving at ultracold velocities, by the time the atom gets to a distance close enough for chemical reaction, it has spontaneously decayed back to the ground state. This is illustrated in Fig. 6, where the relative detuning of the main MOT cooling laser is plotted versus atom-ion separation, assuming a one-linewidth bare-atom detuning as is typical for laser cooling. Also shown in this graph as colored bars are the average distances travelled by $\mathrm{Ca}$ (blue) and $\mathrm{Rb}$ (red) atoms during a collision with a $\sim 150 \mathrm{amu}$ ion at $10 \mathrm{mK}$. Due to the shorter lifetime of the excited state and nature of the Stark shift, the suppression of collisions on excited state channels is much stronger in $\mathrm{Ca}$ than $\mathrm{Rb}$; in general, one would expect, for these reasons, that all alkaline earth MOTs exhibit stronger

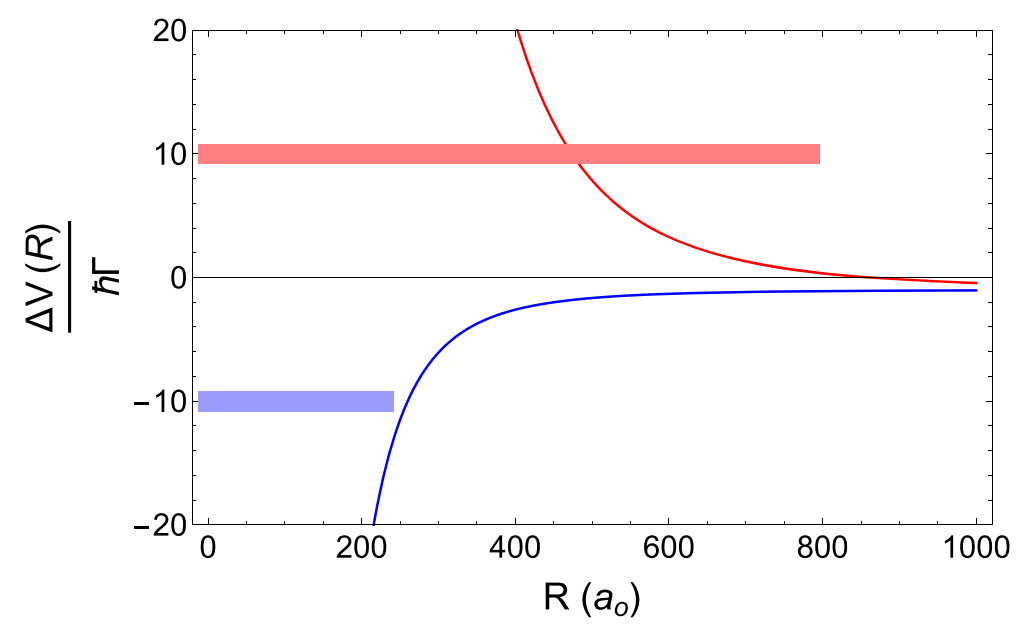

Fig. 6 Detuning of the cooling laser frequency for Ca (blue line) and $\mathrm{Rb}$ (red line) versus atom-ion separation. The lines in this figure assume the lasers are detuned by one linewidth below the bare-atom transition frequency.The ground-state polarizability of the Ca $(\mathrm{Rb})$ atom is larger (smaller) than the excited state, leading to blue-shifting (red-shifting) of the transition frequency as the ion approaches. The blue (red) bars show the distance a $\mathrm{Ca}(\mathrm{Rb})$ atom would travel during a collision with a $\sim 150$ amu ion at $10 \mathrm{mK}$ during one excited state lifetime 
suppression of excited atom collisions than alkali MOTs. In fact, in our work with Ca we find that reactions involving the excited state are suppressed by $\gtrsim 100 \times$ from their 'true' reaction rates and are barely measurable. While in the work of the Basel group, excited state $\mathrm{Rb}$ reactions have been observed in at least two systems $[18,44]$ - we estimate that, depending on parameters, the suppression of Rb excited state collisions is $\sim 1-10 \times$ in these systems and could affect comparison with accurate theoretical calculations.

Finally, it is often useful to have a laser-cooled ion present during experiments in a MOTion trap to enable motional sympathetic cooling, trap imaging, and positioning of molecular ions. It is clearly desirable for the atomic ion used to be immune to reaction with the ultracold atom. This can be achieved for ground state reactions in some cases, e.g. $\mathrm{Ca}+\mathrm{Ba}^{+}$and $\mathrm{Ca}+\mathrm{Sr}^{+}$, but, like the atom-molecular ion case, excited state reactions are typically energetically allowed. While reactions of excited state atoms with the ions are mitigated by the aforementioned Stark shift of the resonant frequency, reactions of ground state atoms with excited state ions do readily occur. This is because excitation of the ion only changes the van der Waals interaction between the atom and ion, which does not significantly perturb the ionic transition frequency until relatively small atom-ion separation. Therefore, ionic excitation can generally survive to the close range necessary for chemistry. Nonetheless, by controlling the ionic excitation to be as small as possible one can reduce the observed reaction rates by $\sim 10 \times$ for species where ground state reactions are precluded, e.g. $\mathrm{Ca}+\mathrm{Ba}^{+}[45]$.

In summary, molecular ions and laser-coolable atoms are by nature reactive. Nonetheless, with some forethought, combinations can be found that have endoergic ground-state reactions and are therefore unable to react at low temperature. Since reactions involving atomic excitation are suppressed, at least in the case of alkaline earth atoms, it appears, at present, that considering the atomic ground-state reaction energetics is sufficient. Currently, the greatest complication for understanding atom-molecular ion chemistry is a paucity of data for the properties of most molecular ions. In these cases, and especially for light molecular ions, quantum chemistry software can be used to estimate the relevant reaction energetics and allow a judicious choice of atom-molecular ion pair.

\section{MOT produced ions}

As with any experimental system, there are a handful of unwanted effects in the MOTion trap that must be controlled. Many of these effects, such as excess micromotion and coherent population trapping in the presence of the MOT field, are handled by standard techniques in the field [46, 47]. One effect, which is particularly peculiar to the MOTion trap, and merits further discussion is the production of unwanted ions by the MOT.

The MOT localizes a high-density sample of atoms in the presence of a large number of optical photons. There are therefore several ways to produce ions from the ultracold atoms. First, the atoms may be multi-photon ionized. Column 4 of Table 2 shows the number of laser-cooling photons required to ionize the atom. All atoms that require less than two photons to be ionized - $\mathrm{Zn}, \mathrm{Be}, \mathrm{Cd}, \mathrm{Mg}$, and $\mathrm{Ho}-$ will produce a significant number of atomic ions since the two-photon ionization process is obviously resonantly enhanced. This process is so severe that in the case of $\mathrm{Cd}$ it has been observed to limit the MOT lifetime [48]. In these cases, one would expect that the MOT produces $>10^{4}$ ions/s and these ions, regardless of their stability in the ion trap, would heat and overwhelm any ions deliberately loaded into the trap. Further, even if direct two-photon ionization by the 
laser cooling photons is precluded, it may still be possible that a large number of atomic ions can be produced if any level in the laser-cooling scheme can be ionized by any laser in the MOTion trap. For example, in a Ca MOT the upper repump state (Ca $\left.4 \mathrm{~s} 5 \mathrm{p},{ }^{1} \mathrm{P}_{1}\right)$ can be ionized by the lasers used to cool $\mathrm{Yb}^{+}$ions.

In instances where two-photon ionization is energetically precluded, it is still possible to produce a significant number of ions in the MOT through photoassociative ionization (PAI) $[49,50]$. PAI produces molecular dimer ions from the MOT atoms through excitation, followed by autoionization or photoassociation directly to a molecular ion state. It is most likely to occur in those atoms that have an ionization potential slightly above the energy of two laser cooling photons. In these cases, the doubly excited state of the neutral dimer molecule is close in energy to the ground state of the molecular dimer ion, which allows for efficient direct and/or auto- ionization. PAI was first observed in $\mathrm{Na}$ [49] with the development of the MOT technique. During the development of the MOTion trap we found that operation of the MOT produced a significant number of $\mathrm{Ca}_{2}^{+}$molecular ions via PAI [51]. To our knowledge, PAI has only been observed in Na and Ca MOTs to date (it has also been observed in a dual-species $\mathrm{Na}$-Cs system [52]), but we expect this is because most MOT experiments do not have the means to detect ions produced in the MOT, and in fact PAI occurs in many MOT experiments. While a calculation of the molecular structure is required to fully ascertain the possibility of PAI in each system, we expect that it occurs to varying degrees in, at least, $\mathrm{Yb}, \mathrm{Tm}, \mathrm{Dy}, \mathrm{Sr}$, and Ba MOTs.

While these ion-production channels lead to atom loss and therefore degrade the performance of the MOT, their most detrimental effect is on the trapped ions. Because these ions are produced at random locations in the trap, they can lead to large heat loads on the ions and, in some cases, loss of the desired molecular ions from the trap. We have found that this effect can be mitigated, in Ca at least, by operating the ion trap in a regime where $\mathrm{Ca}^{+}$and/or $\mathrm{Ca}_{2}^{+}$are not stable - note this may not always be possible since the requisite stability of the desired molecular ion may preclude it. In this regime, the ions are created and then immediately ejected from the trap with minimal effect on the trapped ions. The Smith group at UCONN has also implemented secular frequency excitation to remove unwanted $\mathrm{Na}_{2}^{+}$ions, produced through PAI, from their experiments [53]. It may also be possible to use an intercombination MOT, in e.g. Sr, to produce an ultracold alkaline earth-gas without producing ions, though the reactivity of these long-lived excited states may become problematic.

\section{Generation and detection of molecular ions}

A prerequisite for all of these experiments is the ability to produce and detect the molecular ions of interest. Over the last few years several techniques have emerged as the consensus methods of the field for meeting these requirements. These techniques, and their variations, are briefly described below for completeness.

\section{Generation of molecular ions}

The thermodynamic conditions where ions exists are generally quite extreme compared to our everyday experience. Therefore, typically neutral species must be ionized through the addition of energy in some form. This is accomplished in a variety of ways, with the three most common methods being ionization of neutral molecules in the trap volume, laser ablation, and chemical reaction with trapped atomic ions. 
In the first method, a low-density neutral gas containing a parent molecule is leaked into the vacuum chamber, either through a leak valve or pulsed valve. An electron beam or laser (lasers if a multi-photon ionization scheme is used), is focused into the center of the trapping region. The neutral molecules are then ionized (and perhaps dissociated), producing the desired molecular ion inside the trap volume. This technique, which is reviewed in [54], has many advantages including control of trapped ion number and the possibility for producing rovibrationally state-selected molecular ions.

Another method for producing molecular ions is via laser ablation of a solid target. Here, a solid target (with requisite low vapor pressure) is placed inside the vacuum vessel close to the ion trap. A high-power laser, typically a pulsed Nd:YAG laser, is focused onto the target. The laser dramatically heats a small volume of the target emitting a highlyionized plume of gas from the surface, which contains a large number of atomic and molecular ions derived the target constituents. These ions can be loaded into the ion trap in a number of ways. First, as was demonstrated in [55] the plasma itself can short-circuit the trap electrodes causing a sag in the trapping voltages. By the time the voltages recover, low energy ions from the ablation plume have moved into the trap volume and the trap potential recovers around them. In our experience, we have found that more reproducible loading can be achieved if the trap voltages are instead turned on at a specified delay, typically a few tens of microseconds, following the ablation pulse. Loading stability is further enhanced by ensuring that the phase of the $\mathrm{rf}$ is stable with respect to this turn on, i.e. the $\mathrm{rf}$ is increasing with the same polarity each time it is turned on. While laser ablation is a very simple method for producing a large number of molecular ions, it has the disadvantage that if the plume is not controlled it can lead to patch charging of the electrodes over time; resulting in constantly changing micromotion compensation conditions. Further, the laser ablation process typically leads to a trapped sample composed of many types of ions, e.g. atomic ions and molecular ions species other than the molecular ion of interest. In our experience, we have found that these ions can typically be ejected from the trap by changing the Mathieu $a$ and $q$ parameters to eject the unwanted ions. Secular excitation has been used in other experiments [53] to remove unwanted ions, but it often has the undesirable effect that the excited ions heat and eject the other ions in the trap.

Finally, it is also possible to load molecular ions into the trap by first trapping an atomic ion of interest, usually produced by ionization of a neutral atomic gas, and then leaking in neutral molecular gas to react with the atomic ions and produce the desired molecular ions. This technique was pioneered by the Drewsen group for the production of $\mathrm{MgH}^{+}$ via the reaction of $\mathrm{Mg}^{+}$with $\mathrm{H}_{2}$ [56]. More recently, the Brown group has used a similar technique to produce $\mathrm{CaH}^{+}$[57] and the Chapman group has produced a detailed study on the production of Ba containing molecular ions with this method [58]. The advantage of this technique is that it only requires ionization of a neutral atom, which is typically well known. Further, if the precursor atomic ion is laser cooled then the growth of the molecular ion fraction in the trap can be monitored and precisely controlled to produce a controlled number of molecular ions.

\section{Detection ToF-MS and spectroscopy}

Because the ion trap is largely species independent it is possible to simultaneously trap a variety of atomic and molecular ions. This can be a significant advantage if experiments have the ability to assay the contents of the ion trap. This allows, for example, the careful 
determination of chemical reaction branching ratios and the use of action spectroscopy techniques to record high-resolution spectra with only a few trapped particles. There exist a variety of techniques for assaying the contents of an ion trap, including secular excitation [59] and $q$-scan [60] mass spectrometry. In our limited experience, we have found that these techniques can be sensitive to many systematic effects, especially in systems not explicitly optimized for mass spectrometry, and often do not provide an absolute (or at least easily-calibrated) mass spectrum. (It appears most of these non-idealities stem from the fact that, due to the requirement of optical access, the trapping fields used in hybrid trap experiments are not truly quadrupolar. If more sophisticated trapping geometries are devised that allow optical access, while retaining a truly quadrupolar field these techniques may become useful to the field). To overcome these limitations, we have developed a technique, where ions are radially ejected from a linear ion trap, similar to that proposed in [61], into a time-of-flight mass spectrometer (ToFMS) [62]. Further, if a laser-cooled atomic ion, which sympathetically cools all the trapped ions, is also present in the trap then the mass spectrum is dramatically improved; in fact, we have demonstrated mass resolutions $m / \Delta m>1000$ with a flight distance of only $\sim 30 \mathrm{~cm}[63,64]$.

A detailed review of this technique is provided in [65]. Briefly, by radially ejecting the ions from the trap, instead of ejecting along the axial direction as is usually done, the resolution of the mass spectrometer is improved because the ion cloud is much smaller in this radial dimension. Further, co-trapped laser-cooled atomic ions sympathetically cool all the ions, increasing the assay phase space density and thus the mass resolution. This resolution is sufficient to observe the individual isotopes of the trapped molecular ions and has allowed isotopically resolved chemistry and spectroscopy. Several other groups have also recently developed ToFMS capabilities using variations of the technique [66-69]. In addition to being a powerful measurement capability, we have found that in day-to-day operation the ToFMS provides an incredibly useful diagnostic tool. As a result, all of our current ion trapping systems, regardless of their purpose, include ToFMS capabilities.

\section{Conclusions}

Roughly six years ago the first experiments on producing cold molecular ions via sympathetic cooling began. Since then a variety of experimental challenges, detailed in this review, have been addressed. The original goal of sympathetic cooling of a molecular ion has been demonstrated at the proof-of-principle level [19, 41, 70]. Further, these same systems, perhaps surprisingly, have also shed light on other important areas including the resolution of several longstanding problems in ion trap collision kinematics and non-equilibrium physics [25, 27-29] and the first observations of cold atom-ion chemistry [7].

Currently, the field is poised to go beyond these initial first steps in all three of these areas. Experiments to probe the quantum coherence of the cold molecular ions are underway [71] and represent the first steps towards using molecular ions in quantum applications. Work is also ongoing to use the MOTion trap to study and simulate important nonequlibrium physics phenomenon. Nonequilibrium physics is ubiquitous in a variety of fields, including biology, but there are virtually no model systems, where parameters are precisely known and controllable, to test theoretical predictions. We believe the MOTion trap provides such a system, as detailed here. Further, work studying cold atom-ion chemistry, which has implications in atmospheric, interstellar, and combustion 
chemistry, is becoming more mature. A variety of species have now been studied and theoretical calculations are beginning to be performed. We expect to move from simply studying these reactions to actively controlling them at the quantum level in the next few years. Thus, though still in its infancy, the hope is that further development and use of the MOTion trap system will continue to push our understanding in these diverse fields and make the technologies they promise possible.

\begin{abstract}
Acknowledgements
The author thanks the colleagues, postdoctoral researchers, and graduate students who have made the MOTion trap possible - Alex Dunning, Kuang Chen, Mike Mills, Prateek Puri, Wade Rellergert, Christian Schneider, Steven Schowalter and Scott Sullivan. This review simply repeats the important things they have discovered in their development of the MOTion trap. He also acknowledges a fruitful collaboration with Svetlana Kotochigova on virtually every aspect of his work. This work was supported by National Science Foundation (PHY-1205311) and Army Research Office (W911NF-15-1-0121 and W911NF-14-1-0378) grants.
\end{abstract}

\title{
Competing interests
}

The author declares that he has no competing interests.

Received: 30 August 2016 Accepted: 4 November 2016

Published online: 07 December 2016

\section{References}

1. Haroche S (2008) Essay: Fifty years of atomic, molecular and optical physics in physical review letters. Phys Rev Lett 101:160001

2. Ladd TD (2010) Quantum computers. Nature 464:45

3. Cronin AD, Schmiedmayer J, Pritchard DE (2009) Optics and interferometry with atoms and molecules. Rev Mod Phys 81:1051

4. Ludlow AD, et al (2015) Optical atomic clocks. Rev Mod Phys 87:637

5. Budker D, Romalis M (2007) Optical magnetometry. Nat Phys 3:227

6. Phillips WD (1998) Laser cooling and trapping of neutral atoms. Rev Mod Phys 70:721

7. Dulieu O, Krems R, Weidemueller M, Willitsch S (2011) Physics and chemistry of cold molecules. Phys Chem Chem Phys 13(42):18703-18704

8. Carr LD, DeMille D, Krems RV, Ye J (2009) Cold and ultracold molecules: science, technology and applications. New J Phys 11:055049

9. Barry JF, et al (2014) Magneto-optical trapping of a diatomic molecules. Nature 512:286

10. Yeo M, et al (2015) Rotational state microwave mixing for laser cooling of complex diatomic molecules. Phys Rev Lett 114:223003

11. Kozyryev I, et al (2016) Radiation pressure force from optical cycling on a polyatomic molecules. arXiv:1603.04089

12. Makarov, OP, et al (2003) Radiative charge-transfer lifetime of excited state NaCa+ . Phys Rev A 67:042705

13. Hudson ER (2009) Method for producing ultracold molecular ions. Phys Rev A 79:032716

14. Lim J, et al (2015) Modeling sympathetic cooling of molecules by ultracold atoms. Phys Rev A 92:053419

15. Staanum PF, et al (2010) Rotational laser cooling of vibrationally and translationally cold molecular ions. Nat Phys $6: 271$

16. Schneider T, et al (2010) All-optical preparation of molecular ions in the rovibrational ground state. Nat Phys 6:275

17. Lien CY, et al (2014) Broadband optical cooling of molecular rotors from room temperature to the ground state. Nat Comm 5:4783

18. Hall FJ, Willitsch S (2012) Millikelvin reactive collisions between sympathetically cooled molecular ions and laser-cooled atoms in an ion-atom hybrid trap. Phys Rev Lett 109:233202

19. Hauser D, et al (2015) Rotational state-changing collisions of hydroxyl ions with helium. Nat Phys 11:467

20. March RE (2009) Quadrupole ion traps. Mass Spectrom Rev 28:961

21. Adams CS, Riis E (1997) Laser cooling and trapping of neutral atoms. Prog Quant Electr 21:1

22. Eberle P, et al (2015) lon-atom and ion-molecule hybrid systems: Ion-neutral chemistry at ultralow energies. J Phys Conf Ser 635:012012

23. Major F, Dehmelt H (1968) Exchange-collision technique for the rf spectroscopy of stored ions. Phys Rev 170:91

24. Moriwaki Y, et al (1992) Effect of a heavy collision partner on ion loss from a radio frequency trap. Jpn J Appl Phys $31: 1640$

25. DeVoe R (2009) Power-law distributions for a trapped ion interacting with a classical buffer gas. Phys Rev Lett 102:063001

26. Cetina M, Grier AT, Vuletic V (2012) Micromotion-induced limit to atom-ion sympathetic cooling in paul traps. Phys Rev Lett 109:253201

27. Chen K, et al (2013) Measurement of the Coulomb logarithm in a radio-frequency Paul trap. Phys Rev Lett 110:173003

28. Chen K, et al (2014) Neutral gas sympathetic cooling of an ion in a Paul trap. Phys Rev Lett 112:143009

29. Schowalter SJ, et al (2016) Blue-sky bifurcation of ion energies and the limits of neutral-gas sympathetic cooling of trapped ions. Nat Comm 7:12448

30. Krych M, Idziaszek Z (2015) Description of ion motion in a Paul trap immersed in a cold atomic gas. Phys Rev A 91:023430

31. Tsikata E, et al (2010) Magnetic trapping of NH molecules with 20 s lifetimes. New J Phys 12:065028 
32. Abramowitz M, Stegun IA (1964) Handbook of mathematical functions. National Bureau of Standards, Washington, D.C.

33. de Hoffmann E, Stroobant V (2001) Mass spectrometry: principles and applications, second edition. John Wiley and Sons, Inc., West Sussex

34. Meir Z, et al (2016) Dynamics of a ground-state cooled ion colliding with ultra-cold atoms. arXiv:1603.01810v2

35. Höltkemeier B, et al (2016) Buffer-gas cooling of a single ion in a multipole radio frequency trap beyond the critical mass ratio. Phys Rev Lett 116:233003

36. Goodman DS, et al (2012) lon-neutral sympathetic cooling in a hybrid linear if Paul and magneto-optical trap. Phys Rev A 86:033408

37. Strogatz SH (1994) Nonlinear Dynamics and Chaos: With Applications to Physics, Biology, Chemistry, and Engineering (Studies in Nonlinearity). Perseus Books, Jackson

38. Zipkes C, et al (2011) Kinetics of a single trapped ion in an ultracold buffer gas. New J Phys 13:053020

39. Rouse I, Willitsch S (2015) Superstatistical velocity distributions of cold trapped ions in molecular-dynamics simulations. Phys Rev A 92:053420

40. Campbell WC, et al (2008) Time - domain measurement of spontaneous vibrational decay of magnetically trapped NH. Phys Rev Lett 100:083003

41. Rellergert WG, et al (2013) Evidence for sympathetic vibrational cooling of translationally cold molecules. Nature 495:490

42. Stoecklin T, et al (2016) Explanation of efficient quenching of molecular ion vibrational motion by ultracold atoms. Nat Comm 7:11234

43. Kingdon JB (1995) Landau-zener charge exchange calculations: reactions between $\mathrm{H}$ and opacity project ions. Mon Not R Astron Soc 274:425

44. Hall FJ, et al (2011) Light-assisted ion-neutral reactive processes in the cold regime: Radiative molecule formation versus charge exchange. Phys Rev Lett 107:243202

45. Sullivan ST, et al (2012) Role of electronic excitations in ground-state-forbidden inelastic collisions between ultracold atoms and ions. Phys Rev Lett 109:223002

46. Berkeland DJ, et al (1998) Minimization of ion micromotion in a Paul trap. J Appl Phys 83:5025

47. Berkeland DJ, Boshier MG (2002) Destabilization of dark states and optical spectroscopy in Zeeman-degenerate atomic systems. Phys Rev A 65:033413

48. Brickman KA, et al (2007) Magneto-optical trapping of cadmium. Phys Rev A 76:043411

49. Lett PD, et al (1991) Laser modification of ultracold collisions: Experiment. Phys Rev Lett 67:2139

50. Bagnato VS, et al (1993) Ultracold photoassociative ionization collisions in a magneto-optical trap: The optical-field-intensity dependence in a radiatively dissipative environment. Phys Rev A 48:2523

51. Sullivan ST, et al (2011) Trapping molecular ions formed via photo-associative ionization of ultracold atoms. Phys Chem Chem Phys 13:18859

52. Shaffer JP, Chalupczak W, Bigelow NP (1999) Photoassociative ionization of heteronuclear molecules in a novel two-species magneto-optical trap. Phys Rev Lett 82:1124

53. Sivarajah I, et al (2013) Off-resonance energy absorption in a linear paul trap due to mass selective resonant quenching. Rev Sci Instrum 84:113101

54. Willitsch S (2012) Coulomb-crystallised molecular ions in traps: methods, applications, prospects. Int Rev Phys Chem 31:175

55. Hashimoto Y, et al (2006) Trapping laser ablated Ca+ ions in linear Paul trap. Jap J App Phys 45:7108

56. Mølhave K, Drewsen M (2000) Formation of translationally cold $\mathrm{MgH}^{+}$and $\mathrm{MgD}^{+}$molecules in an ion trap. Phys Rev A 62:011401

57. Khanyile NB, Shu G, Brown KR (2015) Observation of vibrational overtones by single-molecule resonant photo-dissociation. Nat Comm 6:7825

58. DePalatis MV, Chapman MS (2013) Production of translationally cold barium monohalide ions. Phys Rev A 88:023403

59. Baba T, Waki I (1996) Cooling and mass-analysis of molecules using laser-cooled atoms. Jpn J Appl Phys 35:1134

60. Douglas DJ, Frank AJ, Mao D (2005) Linear ion traps in mass spectrometry. Mass Spectrom Rev 24:1

61. Denisov EV, Kholomeev A, Makarov AA (2009) RF power supply for a mass spectrometer. Patent 7,498,571

62. Schowalter SJ, et al (2012) An integrated ion trap and time-of-flight mass spectrometer for chemical and photo-reaction dynamics. Rev Sci Instrum 83:043103

63. Schneider C, et al (2014) Laser-cooling-assisted mass spectrometery. Phys Rev App 2:034013

64. Schneider C, et al (2016) Electronics of an ion trap with integrated time-of-flight mass spectrometer. Int J Mass Spec 394:1

65. Schowalter SJ Action spectroscopy of molecular ions and studies of cold collisions in a hybrid atom-ion trap. PhD thesis, University of California Los Angles

66. Seck CM, et al (2014) Rotational state analysis of $\mathrm{AlH}^{+}$by two-photon dissociation. J Mol Spec 300:108

67. Ni KK, et al (2014) State-specific detection of trapped $\mathrm{HfF}^{+}$by photodissociation. J Mol Spec 300:12

68. Deb N, et al (2015) Coulomb crystal mass spectrometry in a digital ion trap. Phys Rev A 91:033408

69. Rösch D, et al (2016) Design and characterization of a linear quadrupole ion trap for high-resolution coulomb-crystal time-of-flight mass spectrometry. EPJ Tech Instr 3:5

70. Hansen D, et al (2014) Efficient rotational cooling of coulomb-cyrstallized molecular ions by a helium buffer gas. Nature 508:76

71. Wolf F, et al (2016) Non-destructive state detection for quantum logic spectroscopy of molecular ions. Nature 530:457

72. Grier AT, et al (2009) Observation of cold collisions between trapped ions and trapped atoms. Phys Rev Lett 102:223201

73. Zipkes $C$, et al (2010) A trapped single ion inside a bose - einstein condensate. Nature 464:388

74. Schmid C, Harter A, Denschlag JH (2010) Dynamics of a cold trapped ion in a Bose-Einstein condensate. Phys Rev Lett 105:133202

75. Hall FHJ, et al (2013) Light-assisted cold chemical reactions of barium ions with rubidium atoms. Mol Phys 111:1683 
76. Rellergert WG, et al (2011) Measurement of a large chemical reaction rate between ultracold closed-shell Ca-40 atoms and open-shell $\mathrm{Yb}^{+}-174$ ions held in a hybrid atom-ion trap. Phys Rev Lett 107:243201

77. Sivarajah I, et al (2012) Evidence of sympathetic cooling of $\mathrm{Na}^{+}$ions by a Na magneto-optical trap in a hybrid trap. Phys Rev A 86:063419

78. Deiglmayr J, et al (2012) Reactive collisions of trapped anions with ultracold atoms. Phys Rev A 86:043438

79. Ravi K, et al (2012) Cooling and stabilization by collisions in a mixed ion - atom system. Nat Comm 3:1126

80. Radzig A, Smirnov B (1985) Reference data on atoms, molecules, and ions. Springer, Berlin

81. Webbook NC. http://webbook.nist.gov/chemistry/

82. Kaledin LA, Heaven MC, Field RW (1999) Thermochemical properties ( $\mathrm{D}_{0}^{o}$ and IP) of the lanthanide monohalides. J Mol Spec 193:285

Submit your manuscript to a SpringerOpen ${ }^{\circ}$ journal and benefit from:

- Convenient online submission

- Rigorous peer review

- Immediate publication on acceptance

- Open access: articles freely available online

- High visibility within the field

- Retaining the copyright to your article

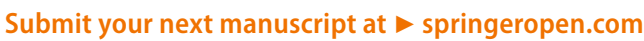

